\title{
Evaluation of a glomerular filtration term in the DISST model to capture the glucose pharmacodynamics of an insulin resistant cohort
}

Paul D Docherty

J Geoffrey Chase

Thomas F Lotz

Jeremy D Krebs 


\section{Study design}

Study: 24 week Atkins-diet based intervention

- Weeks 1-12 weight loss stage. Weeks 13-24 weight maintenance

- Significant carbohydrate limit $\sim 20 \mathrm{~g} /$ day - target energy composition: $20-25 \%$ via carbohydrate; $30 \%$ via protein; and $45-$ $50 \%$ via fats

Participants: 12 individuals with established type $\mathbf{2}$ diabetes

- Duration of diabetes 0.5-12 years

- BMI range $34-46 \mathrm{~kg} / \mathrm{m}^{2}$

- Exclusion criteria: No other major illness or recent weight change 


\section{Study Evaluation}

Tests: Week 0, 12 and 24

- Bodyweight and composition

- Fasting assays - lipids, HbA1c, Creatinine

- Insulin sensitivity (SI) test

SI test protocol: GM Ward et al. (Metabolism 2001 50(5))

- $0.2 \mathrm{~g} / \mathrm{kg}$ Glucose bolus $(\mathrm{t}=0)$

- $3.5 \mathrm{mU} \cdot \mathrm{kg}^{-1} \cdot \mathrm{min}^{-1}$ insulin (0 to 2 ); $0.5 \mathrm{mU} \cdot \mathrm{kg}^{-1} \cdot \mathrm{min}^{-1}$ (7 to 17 ); $0.25 \mathrm{mU} \cdot \mathrm{kg}^{-1} \cdot \mathrm{min}^{-1}$ (17 to 50 ) $0.1 \mathrm{mU} \cdot \mathrm{kg}^{-1} \cdot \mathrm{min}^{-1}$ (50 to 300 )

- 33 samples, 5+ hours - Samples at $\mathrm{t}=-10,-5,-1,0,1,2,3,4,5,6,8,10,12.5$, $15,20,25,30,35,40,50,60,70,80,90,100,120,140,160,180,210,240,270,300$ minutes

- Assayed for glucose and insulin 


\section{S/ test evaluation}

Model Based:

- Standard DISST model (McAuley et al. 2011 Metabolism)

- DISST model modified with a glomerular filtration (GFR) term

- DISTq (Docherty et al. 2009 Open Med. Inf. J. 3)

- Minimal Model

Simple Measures:

- Area Under the Curve $(A \cup C)$ glucose $\left(A \cup C_{G}\right)$, and insulin $\left(A \cup C_{1}\right)$

- HOMA

- Basal assays: glucose $\left(G_{B}\right)$, insulin $\left(I_{B}\right), \mathrm{HbA} 1 \mathrm{c}$ and triglyceride (TAG) 


\section{DISST model}

Exogenous Post hepatic insulin bolus $\beta$-cell secretion
Endogenous glucose Exogenous

production glucose bolus
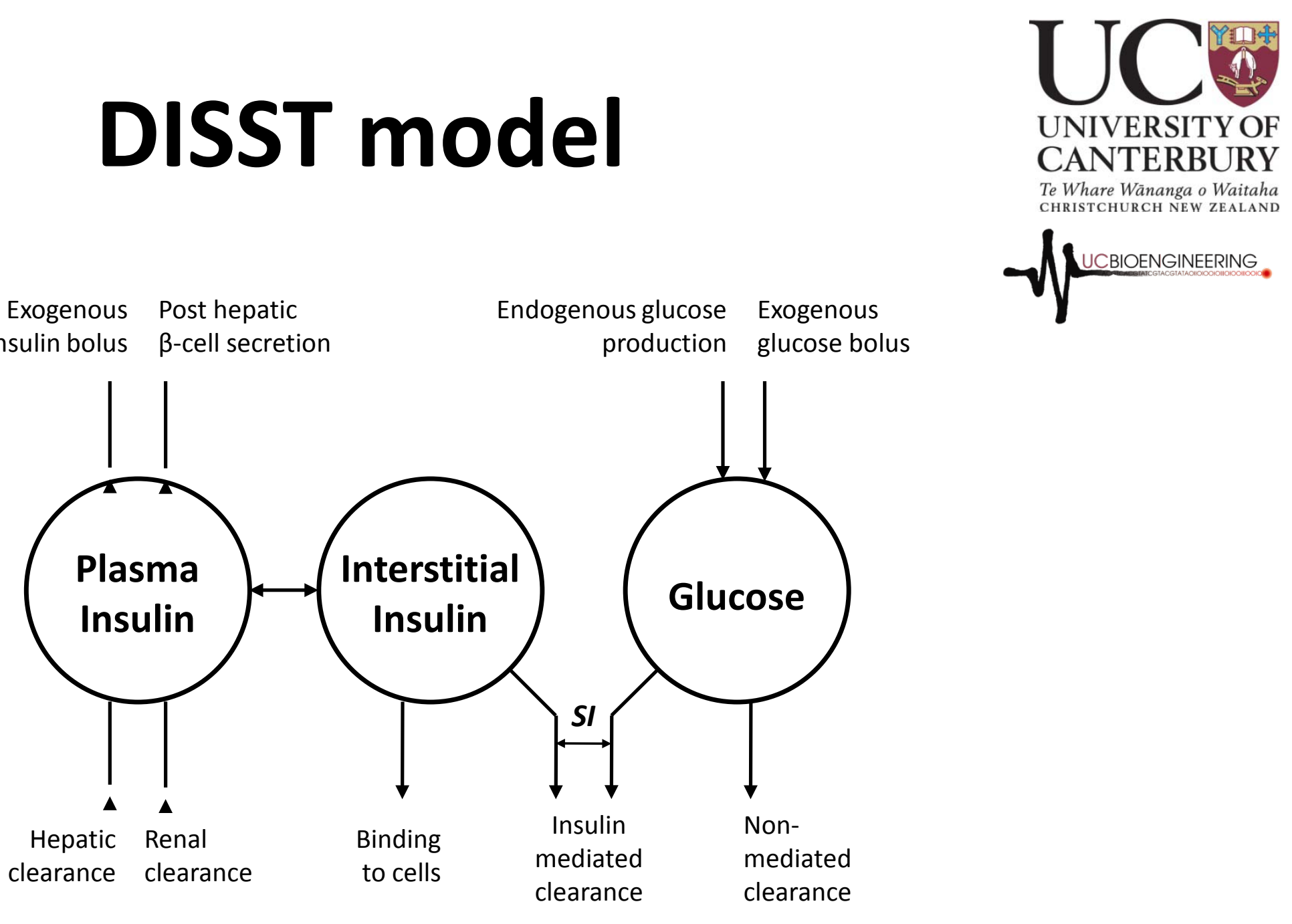

$$
\begin{aligned}
\dot{Q}= & \frac{n_{I}}{V_{Q}} I-\left(n_{C}+\frac{n_{I}}{V_{Q}}\right) Q \\
& \dot{G}=p_{G}\left(G_{0}-G\right)-S I_{\text {DISST }}\left(G Q-G_{0} Q_{0}\right)+\frac{P_{X}}{V_{G}}
\end{aligned}
$$




\section{DISST validation}

- Sparsely-sampled, low-dose, short duration IM-IVGTT protocol

- Concurrent model-based assessment of $S I$ and endogenous insulin production

- High intra-patient repeatability

(11-13\% variation)

- High gold standard correlation $(R=0.81)$, absolute $\left(\Delta_{\text {median }}=-10.6 \%\right)$ and diagnostic equivalence (ROC c-unit=0.96)

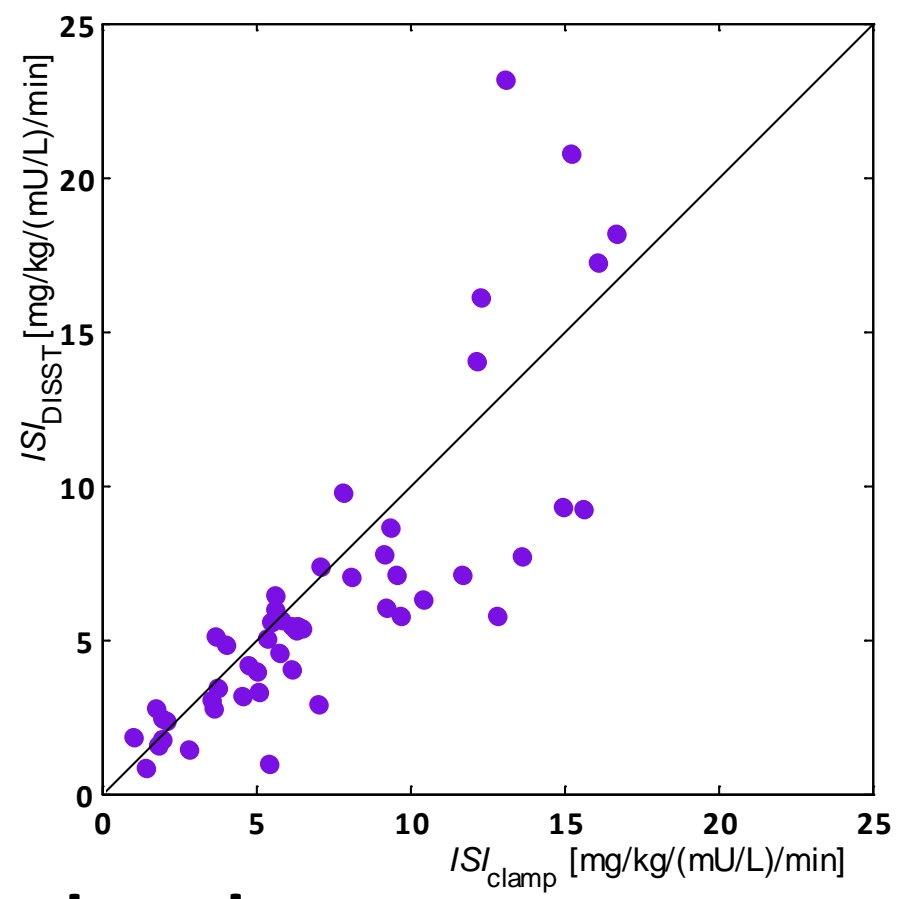

Important note: DISST protocol was not undertaken $\rightarrow$ results of this study not indicative of DISST test 


\section{Glomerular filtration term}

From Arleth et al. (2000 CMPB 62(3))

- Initial trace of clearance at $10 \mathrm{mmol} / \mathrm{L}$ glucose

- Linear clearance at $22 \mathrm{mmol} / \mathrm{L}$ glucose

- Smooth transition

$$
\begin{aligned}
& G F R=\left\{\begin{array}{cc}
0, & G<10 \\
0.077 G^{2}-1.54 G+7.72 & 10<G<22 \\
1.85 G-29.62 & G>22
\end{array}\right\} / 1000 \\
& \dot{G}=p_{G}\left(G_{0}-G\right)-S I_{D I S S T}\left(G Q-G_{0} Q_{0}\right)+\frac{P_{X}-G F R \cdot B W}{V_{G}}
\end{aligned}
$$




\section{Parameter identification}

The iterative integral method (IIM):

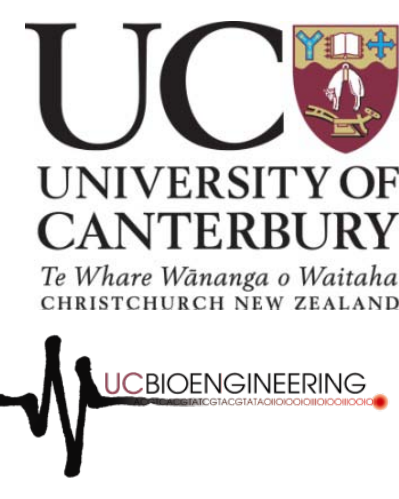

- Robust $\rightarrow$ not dependent on starting estimations

- Does not locate local minima

- Computationally quick $\rightarrow$ recent studies have exhibited a 3 to $350 x$ faster convergence than Levenburg-Marquardt algorithms.

- Does not explicitly map error surface $\rightarrow$ does not become unstable due to over-sized convergence steps

- Docherty et al. published in application (Open Med. Inf. J 2009 3) validation paper in review (Med. Biol. Eng. Comput.) 


\section{IIM method}

1. Simulate species: $G=$ interpolate $G$ data

2. Evaluate eqn coefficients at sample times:

$$
\begin{aligned}
& \text { LHS }=G_{i}-G_{O}+p_{G} \int G(t)-G_{0} \mathrm{dt}_{0 \rightarrow i} \\
& C S I=-\int G(t) Q(t)-G_{0} Q_{0} \mathrm{dt}_{0 \rightarrow i} \\
& C V_{G}=\int P_{X}(t)-G F R(t) * B W \mathrm{dt}_{0 \rightarrow i} \quad \text { where } i \text { is the sample times }
\end{aligned}
$$

3. Evaluate matrix representation:

$$
\left\{\mathrm{LHS}_{0 \rightarrow i}\right\}^{\top}=\left[\mathrm{CSI}_{O \rightarrow i}, C V_{G 0 \rightarrow i}\right]\left\{S I, 1 / V_{G}\right]^{\top}
$$

4. Re-simulate $G(t)$ as function of $S I$ and $V_{G}$ outcomes, re-simulate $G F R(t)$ if relevant

5. Repeat steps 2 to 4 until convergence 


\section{Minimal Model ID}

Minimal model is re-arranged to allow identification with IIM:

- Governing eqns: $\dot{G}=p_{1}\left(G_{0}-G\right)-G X+\frac{P_{X}}{V_{G}}$

$$
\dot{X}=p_{3}\left(I-I_{0}\right)-p_{2} X, \quad S G_{M M}=p_{1}, \quad S I_{M M}=p_{3} / p_{2}
$$

- Can be rearranged: $X=X_{0}+p_{3} \int\left(I-I_{0}\right) d t-p_{2} \int X d t$

$$
\begin{gathered}
\dot{G}=p_{1}\left(G_{0}-G\right)-G p_{3} \int\left(I-I_{0}\right) d t+G p_{2} \int(X) d t+\frac{P_{X}}{V_{G}} \\
G-G_{0}=p_{1} \int\left(G_{0}-G\right) d t-p_{3} \int G \int\left(I-I_{0}\right) d \tau d t+p_{2} \int G \int(X) d \tau d t+\frac{1}{V_{G}} \int P_{X} d t
\end{gathered}
$$

- And enable the matrix formulation using the following

Coefficients: LHS; $C_{n}=G_{n}-G_{0}$, RHS; $P 1_{n}=\int_{0}^{n} G_{0}-G d t$,

$P 2_{n}=\int_{0}^{n} G \int_{0}^{n} X d \tau d t, P 3_{n}=-\int_{0}^{n} G \int_{0}^{n}\left(I-I_{0}\right) d \tau d t$ and $V_{n}=\int_{0}^{n} P_{X} d t$ 


\section{Comparison of IIM and}

\section{Levenberg-Marquardt (L-M)}
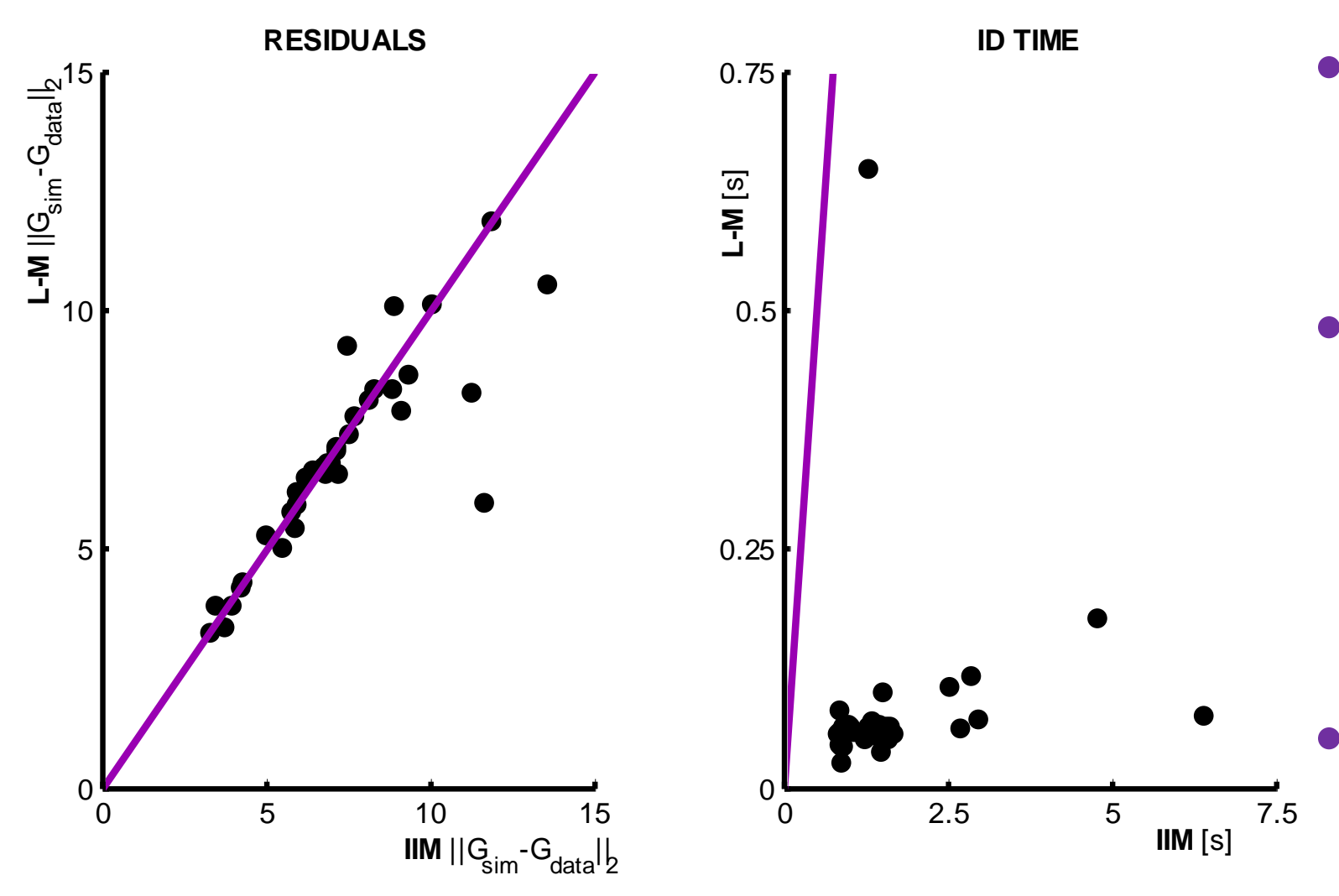

(Minimal model identified using full dataset in IIM and L-M parameter identification methods)

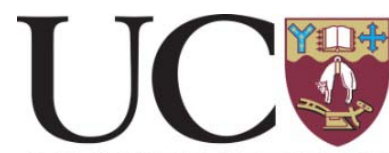

- Residuals of IIM comparable to L-M

- L-M much faster than IIM due to indirect identification of parameters in IIM

- Not ideal application of IIM - but it worked nonetheless! 


\section{Comparison between DISST, DISST+GFR and Minimal model}
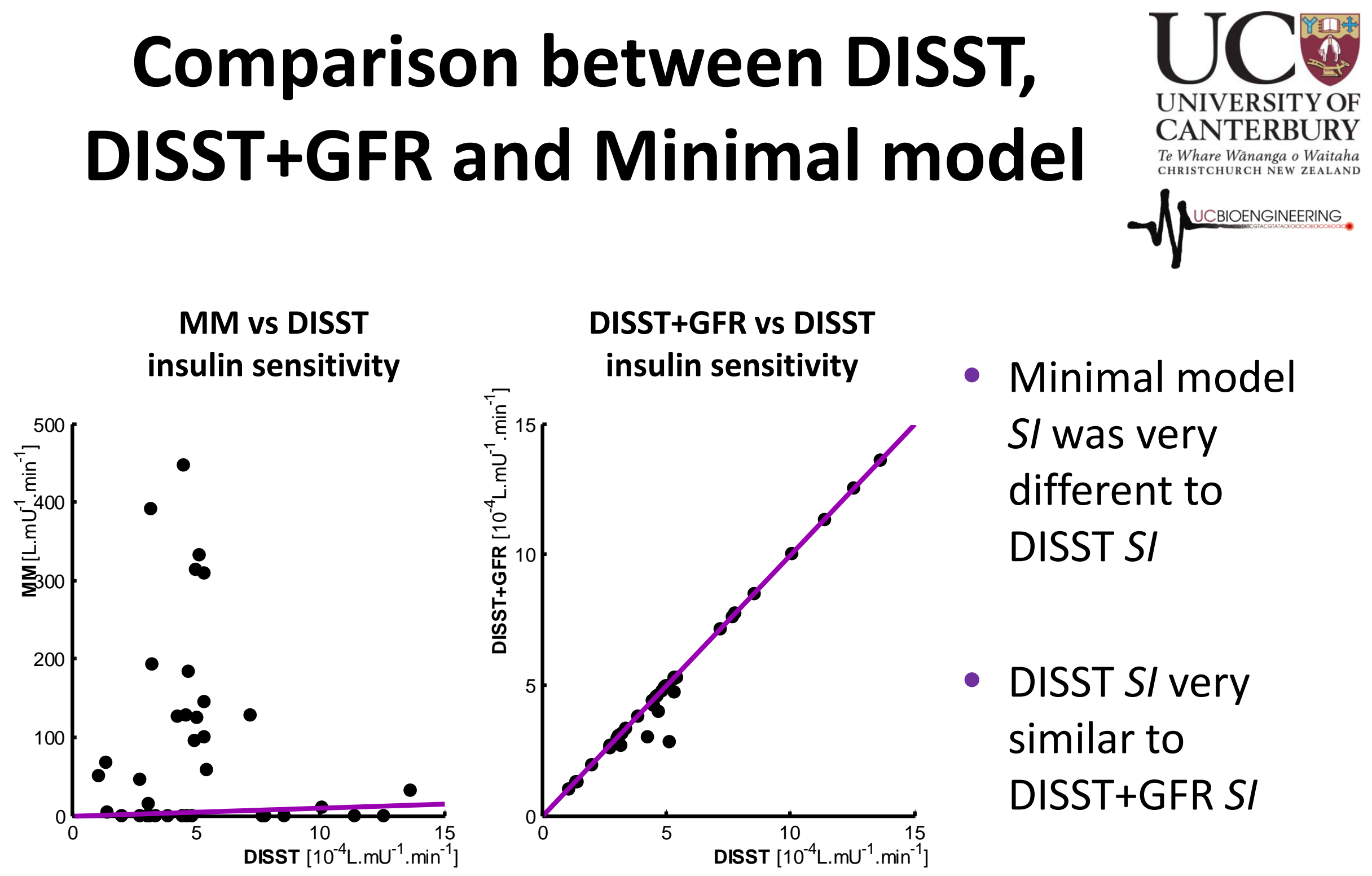

- Minimal model

SI was very different to DISST SI

- DISST SI very similar to DISST+GFR SI 


\section{Residuals}

$\checkmark$ uncBoengineering.
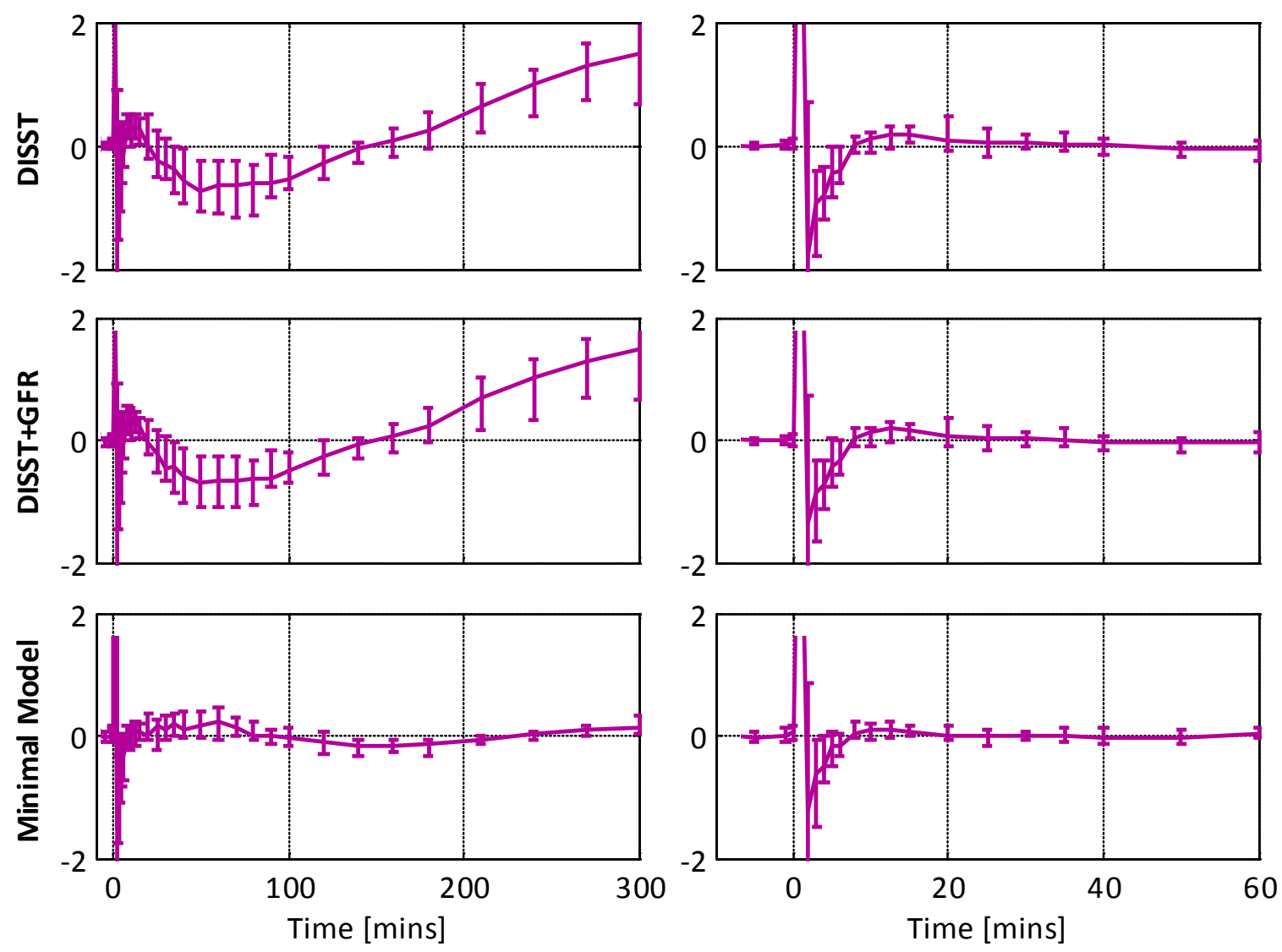

- Minimal model solutions were generally more adherent to the measured data

- DISST and DISST+GFR were not able to characterise 300 minute profile, but were similar to $\mathrm{MM}$ over -10 to 60 mins 


\section{Investigation outcomes}

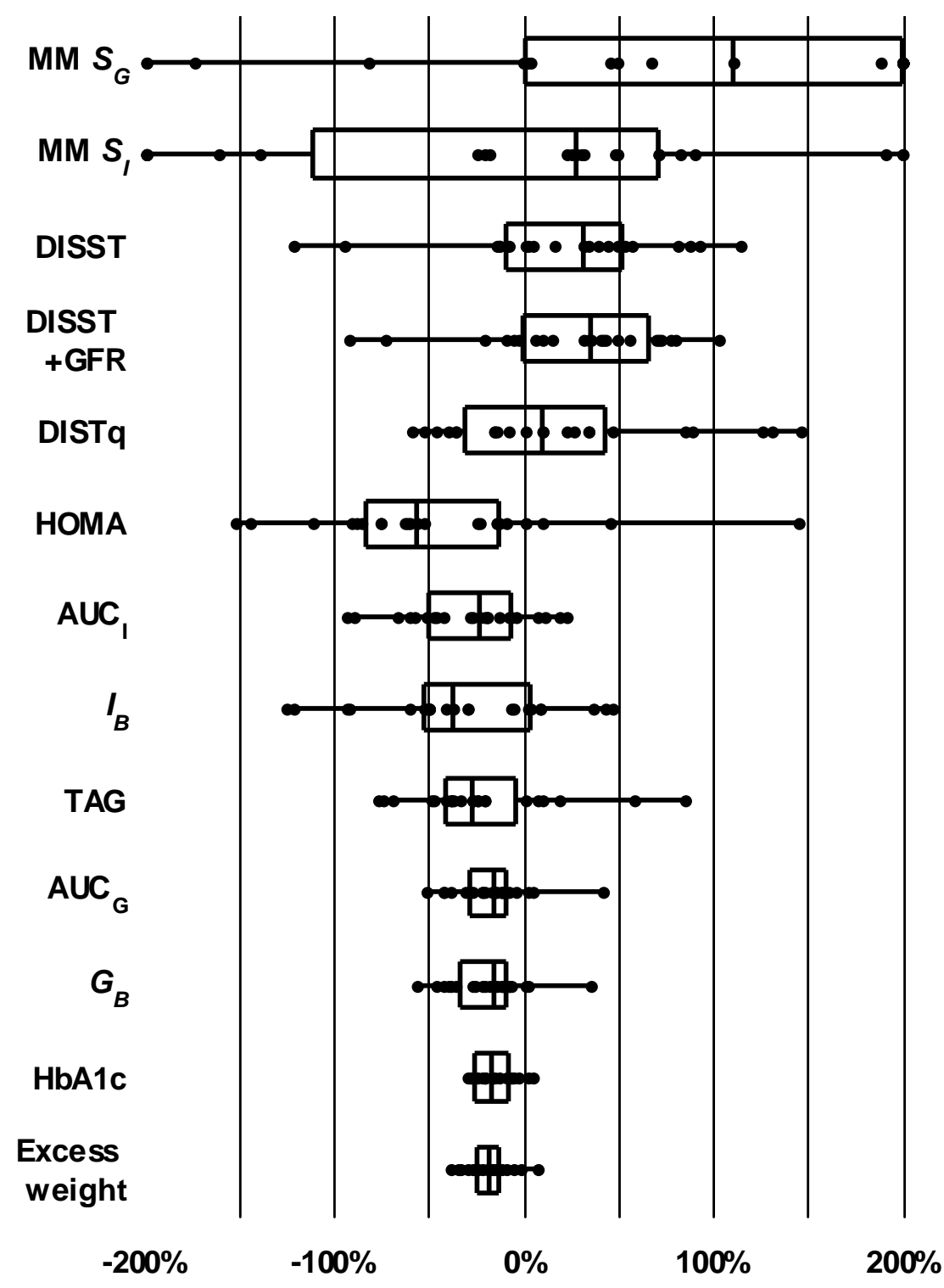

- Compliance to the conditions of the dietary intervention trial was generally good

- Weight loss was observed across the trial 


\section{Model-based outcomes}

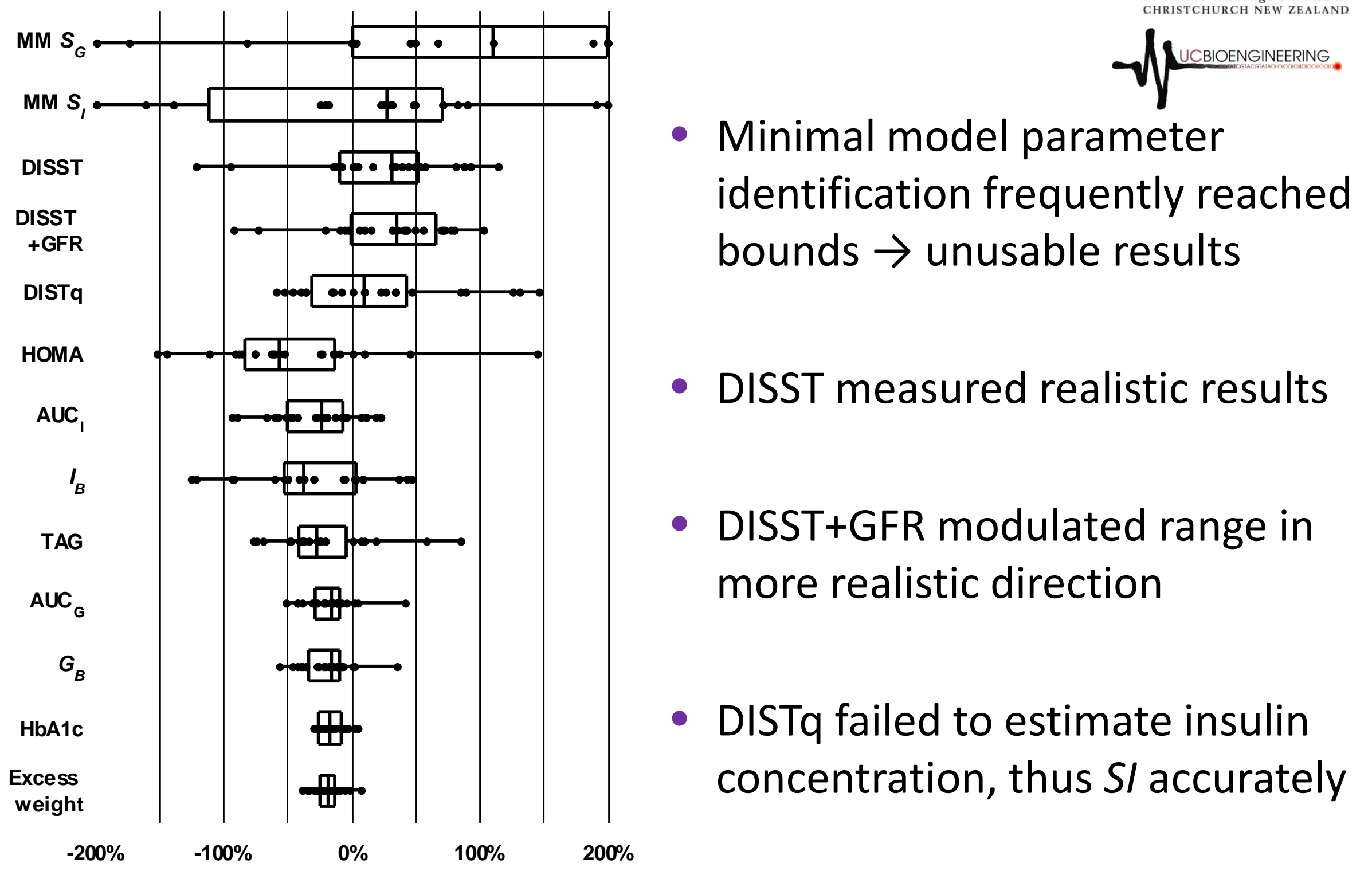




\section{Simple metric outcomes}

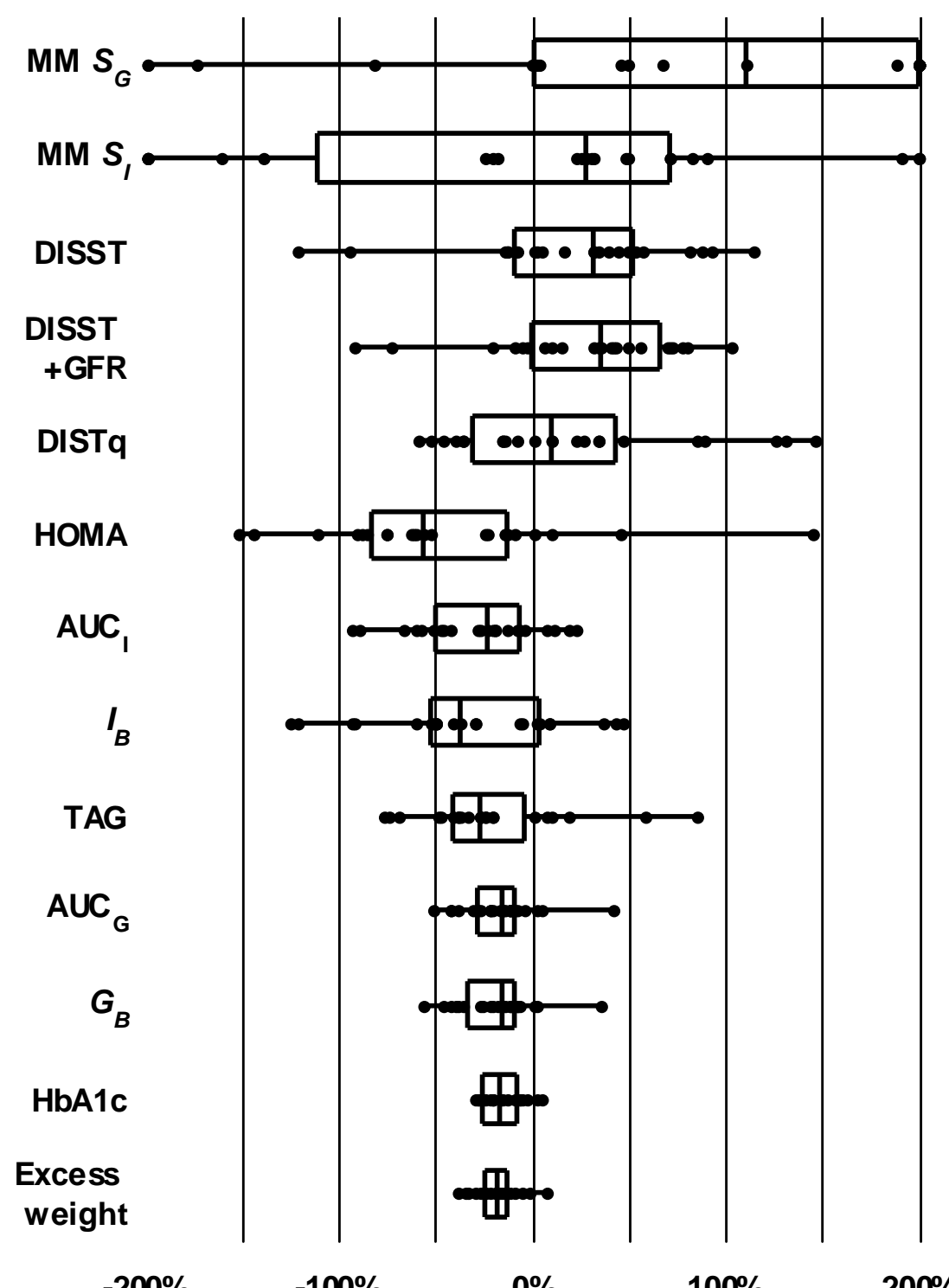

- Fasting assays (HbA1c, $G_{B}, I_{B}$, TAG) generally improved

- $\triangle \mathrm{AUC}_{\mathrm{G}}$ was indistinct to $\Delta G_{B}$ $(\rho=0.90) \rightarrow$ obsolete

- $\triangle \mathrm{AUC} \mathrm{C}_{1}$ was indistinct to $\Delta I_{B}$ $(\rho=0.69) \rightarrow$ obsolete

- $\triangle \mathrm{HOMA}$ similar to $\Delta I_{B}$ $(\rho=0.94)$ 


\section{Study outcomes}

Regarding model-based metrics:

- Minimal model unstable in this IR cohort $\rightarrow$ previously reported

- DISST stable, but unable to characterise longer-term steadystate condition. DISTq stable - but inaccurate.

- Adding variable model parameters can aid residuals but can also obscure results or disable stable parameter estimation

- The addition of the GFR term modulated the glucose clearance in some IFG participants and had a positive contribution to the study outcomes. However the effect was too small to assess 


\section{Study outcomes}

Regarding simple metrics:

- Simple, single blood-test HOMA observed very large, possibly excessive changes

- $\mathrm{HbA1c}, G_{B}, I_{B}$ and weight all exhibited positive outcomes at a cohort level

- $\Delta G_{B}$ described the same changes as $\Delta \mathrm{AUC}_{\mathrm{G}} \rightarrow$ clinical protocol was not necessary if $A \cup C_{G}$ is intended

- $\Delta I_{B}$ described the same changes as $\Delta \mathrm{AUC}_{1} \rightarrow$ clinical protocol was not necessary if $A U C_{1}$ is intended

- TAG exhibited reasonable behaviour but had a significant number of confounders 


\section{Questions?}

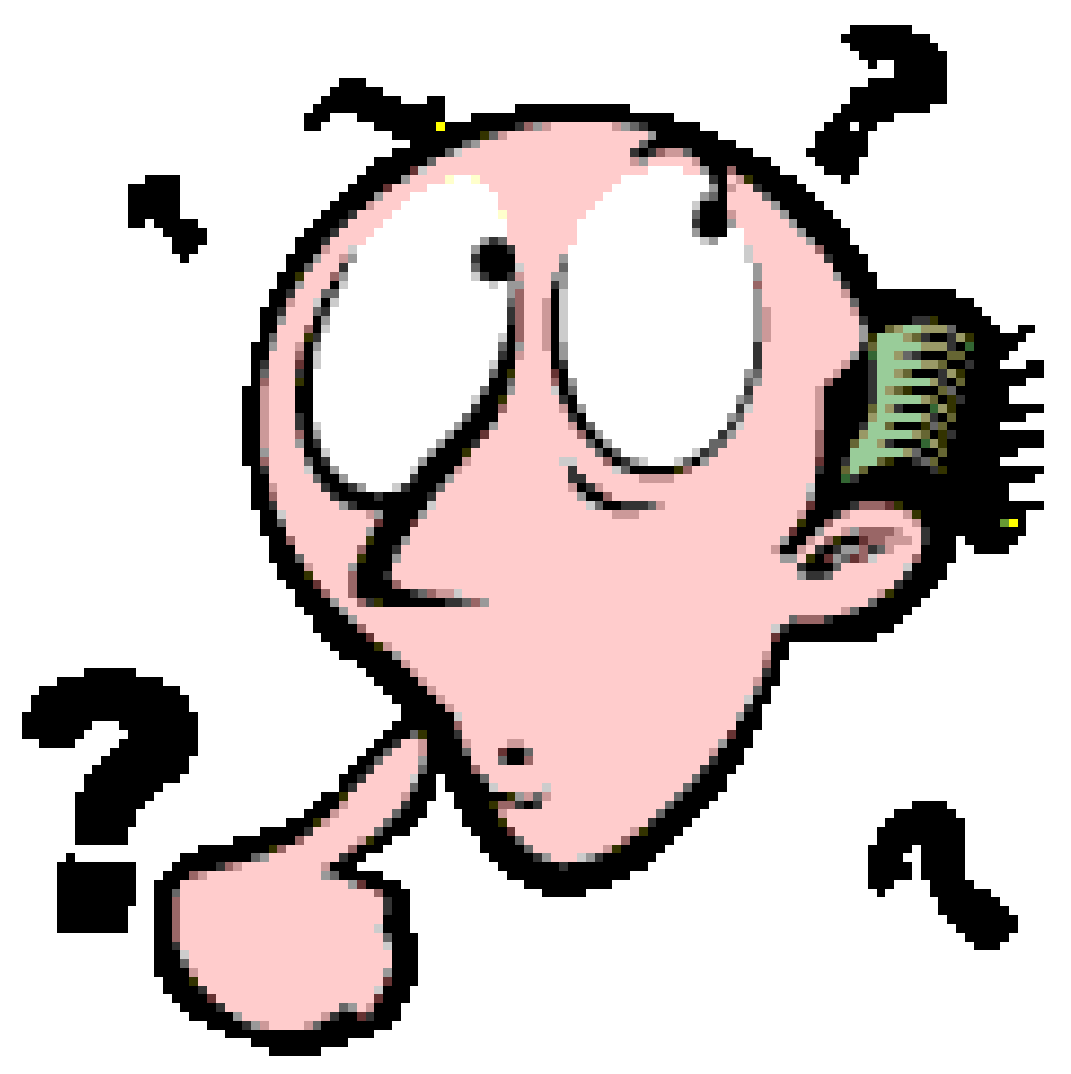

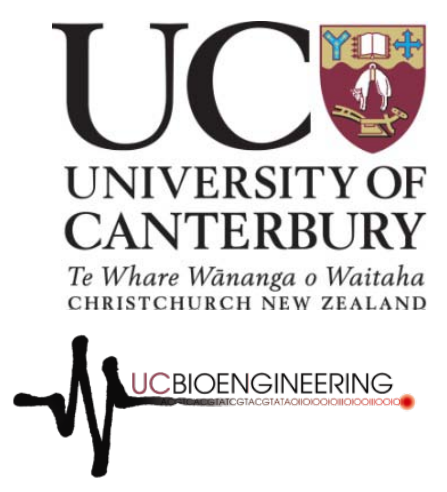

